\title{
Three-loop vertex corrections
}

\author{
Matthias Steinhauser * \\ Karlsruhe Institute of Technology \\ E-mail: matthias.steinhauser@kit.edu
}

We describe recent evaluations of three-loop vertex corrections which have been performed in the context of different physical applications: the massless quark and gluon form factor, the vector current matching coefficient between QCD and NRQCD, and the virtual corrections of the gluonHiggs coupling with finite top quark mass.

RADCOR 2009 - 9th International Symposium on Radiative Corrections (Applications of Quantum Field Theory to Phenomenology)

October 25-30 2009

Ascona, Switzerland

${ }^{*}$ Speaker. 


\section{Three-loop fermion and gluon form factors}

In this Section we consider massless QCD and discuss the virtual corrections to the photonquark and Higgs-boson-gluon vertex (see also Ref. [1] and references therein). It is convenient to decompose the vertex functions according to the Lorentz structure and define the form factors $F_{q}$ and $F_{g}$ via $\Gamma_{q}^{\mu}=\gamma^{\mu} F_{q}\left(q^{2}\right), \Gamma_{g}^{\mu v}=\left(q_{1} \cdot q_{2} g^{\mu v}-q_{1}^{v} q_{2}^{\mu}\right) F_{g}\left(q^{2}\right)$, where $q=q_{1}+q_{2}$ and $q_{1}\left(q_{2}\right)$ is the incoming (anti-)quark momentum in the case of $F_{q}$, and $F_{g}$ depends on the gluon momenta $q_{1}$ and $q_{2}$ with polarization vectors $\varepsilon^{\mu}\left(q_{1}\right)$ and $\varepsilon^{v}\left(q_{2}\right)$. Both for $F_{q}$ and $F_{g}$, which are obtained by applying appropriate projectors, we have $q_{1}^{2}=q_{2}^{2}=0$. Some sample Feynman diagrams contributing to $F_{q}$ and $F_{g}$ are shown in Fig. 1.

For our calculation we have used two different setups. The basic idea of the first one has been described in Refs. [2]: integral representations for the coefficients of the master integrals are derived. They depend on the exponents of the denominators of the integral under consideration and the space-time dimension $d$. In the recent years a procedure has been developed to evaluate the resulting parameter integrals in the limit of large $d$ (see, e.g., Ref. [3]). Knowing sufficiently many expansion terms the coefficient function can be reconstructed since (for fixed exponents) it is a rational function in $d$. The evaluation of the three-loop vertex corrections profited quite a lot from the experience gained in the context of the evaluation of the four-loop two-point functions [4] and the findings of Ref. [5]. In the latter paper it has been shown that the recurrence relations of $n$-loop three-point functions are equivalent to $(n+1)$-loop two-point functions.

The second method has only been applied to the singlet diagrams (see, e.g., Fig. 1(b)) contributing to $F_{q}$. It relies on the idea to combine the Laporta method [6] with the Gröbner bases technique [7] which has been published in the computer code FIRE [8].

We parameterize the results for $F_{q}$ and $F_{g}$ in terms of the bare coupling which allows us to factorize all occurring logarithms of the form $\ln \left(Q^{2} / \mu^{2}\right)$, where $Q^{2}=-q^{2}>0$, and to cast the expressions in the form $(x=q, g)$

$$
F_{x}=1+\sum_{n}\left(\frac{\alpha_{s}}{4 \pi}\right)^{n}\left(\frac{\mu^{2}}{Q^{2}}\right)^{n \varepsilon} F_{x}^{(n)} .
$$

We refrain from listing the results in terms of general SU(3) colour factors, which can be found in Ref. [9], however, we present for illustration the finite ${ }^{1}$ part of $F_{g}$ in the case of QCD where it takes

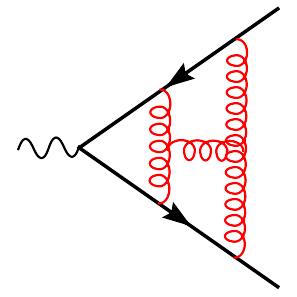

(a)

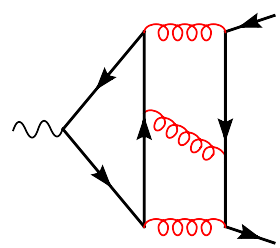

(b)

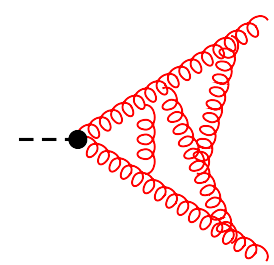

(c)

Figure 1: Sample Feynman diagrams contributing to the $F_{q}\left((\mathrm{a})\right.$ and (b)) and $F_{g}$ (c) at three-loop order. Straight and curly lines denote quarks and gluons, respectively.

\footnotetext{
${ }^{1}$ We refer to Refs. [10] for the divergent contribution.
} 
the form

$$
\begin{aligned}
F_{g}^{(3)}= & \frac{14423912}{243}+\frac{384479 \zeta_{2}}{108}-\frac{370649 \zeta_{3}}{18}+\frac{280069 \zeta_{4}}{32}+\frac{49167 \zeta_{2} \zeta_{3}}{4}-\frac{199263 \zeta_{5}}{10}+\frac{1635 \zeta_{3}^{2}}{4} \\
& -\frac{4527765 \zeta_{6}}{256}-27 X_{9,1}+54 X_{9,2}+n_{f}\left(-\frac{11801309}{1458}-\frac{42296 \zeta_{2}}{81}+\frac{41018 \zeta_{3}}{81}-247 \zeta_{2} \zeta_{3}\right. \\
& \left.+\frac{1055 \zeta_{4}}{8}+\frac{16982 \zeta_{5}}{45}\right)+n_{f}^{2}\left(\frac{2239573}{4374}+\frac{4 \zeta_{2}}{3}+\frac{3376 \zeta_{3}}{81}+\frac{349 \zeta_{4}}{18}\right) .
\end{aligned}
$$

The two constants $X_{9,1}$ and $X_{9,2}$ take the numerical values $X_{9,1} \approx 1428.9963678666183591$ and $X_{9,2} \approx 528.0583 \pm 0.0326$ where $X_{9,1}$ is available analytically [11] and $X_{9,2}$ is known numerically [9, 11] with the indicated precision.

The new NNNLO results for the form factors constitute building blocks for a number of applications. Among them are the virtual corrections to Higgs boson production in gluon fusion, the Drell-Yan process and the two-jet cross section in $e^{+} e^{-}$collisions.

\section{Three-loop matching of the vector current}

In contrast to the previous Section we consider here QCD with $n_{h}=1$ massive and $n_{l}$ massless quarks and evaluate the matching coefficient between QCD and non-relativistic QCD (NRQCD) for the vector current. This quantity is important for phenomena where two heavy quarks are produced in electron-positron annihilation or a bound state of two heavy quarks decays into a lepton pair.

The vector currents in QCD and NRQCD are given by $j_{v}^{\mu}=\bar{Q} \gamma^{\mu} Q$, and $\tilde{j}^{k}=\phi^{\dagger} \sigma^{k} \chi$, where $Q$ denotes a generic heavy quark with mass $m_{Q}$ and $\phi$ and $\chi$ are two-component Pauli spinors for quark and anti-quark, respectively, and $\sigma^{k}(k=1,2,3)$ are the Pauli matrices. The two currents $j_{v}^{\mu}$ and $\tilde{j}^{k}$ can be used to compute vertex corrections with two on-shell quarks and momenta $q_{1}$ and $q_{2}$ $\left(\Gamma_{v}\right.$ and $\tilde{\Gamma}_{v}$ ). From the requirement that the results agree up to power-corrections in $m_{Q}$ defines the matching coefficient $c_{v}$

$$
Z_{2} \Gamma_{v}=c_{v} \tilde{Z}_{2} \tilde{Z}_{v}^{-1} \tilde{\Gamma}_{v}+\ldots
$$

where $Z_{2}$ denotes the on-shell wave function renormalization constant $[12,13]$ and quantities with a tilde are defined within NRQCD. The ellipses in Eq. (2.1) represent terms of order $1 / m_{Q}$ which are neglected.

For the evaluation of $c_{v}$ it is convenient to consider $q^{2}=\left(q_{1}+q_{2}\right)^{2} \approx 4 m_{Q}^{2}$ and apply the so-called threshold expansion [14] to $\Gamma_{v}$ which identifies the hard, soft, potential and ultra-soft integration regions. The latter three contributions are present both on the left- and right-hand side of Eq. (2.1) and thus cancel out. Only the hard contribution where $q^{2}=4 m_{Q}^{2}$ and which is only present in $\Gamma_{v}$ has to be evaluated. This reasoning is based on the use of Dimensional Regularization which is crucial for the evaluation of higher order corrections to $c_{v}$ since scaleless integrals are automatically set to zero. This concerns in particular $\tilde{Z}_{2}$ and $\tilde{\Gamma}_{v}$ which are both identical to one.

The two-loop corrections to $c_{v}$ have been evaluated in Refs. $[15,16,17]$ and the fermionic three-loop contribution in Refs. [18, 19]. The setup used in Ref. [19] is completely automated. It is based on a chain of programs which work hand-in-hand. The starting point is QGRAF [20] 
which generates the amplitudes for each Feynman diagram. In a next step q2e and exp [21] are used in order to identify the topologies and generate input expression in FORM [22] format. The same input file containing the description of the topologies needed by exp is also used in order to provide the input for crusher [23] which performs the reduction of all occurring integrals to a basic set, so-called the master integrals. The topology file is also used for providing the necessary input for FIESTA $[24,25]$ which is employed for the numerical evaluation of all master integrals.

There are several checks on the correctness of our results. As far as the analytical part is concerned the most important one is the evaluation of the Feynman diagrams or general gauge parameter $\xi$ : we have checked that the linear $\xi$-term drops out in our three-loop result. A strong check of the numerical part of our calculation is the change the master integral basis, which is achieved analytically with the help of the integration-by-parts relations generated by crusher. In the new expression, which is a completely different linear combination of master integrals, the numerical evaluation is again performed with the help of FIESTA.

In numerical form the result for $c_{v}$ is given by (for $\mu=m_{Q}$ ):

$$
\begin{aligned}
c_{v}=1 & -2.67 \frac{\alpha_{s}}{\pi}+\left(-44.55+0.41 n_{l}\right)\left(\frac{\alpha_{s}}{\pi}\right)^{2} \\
& +\left(c_{v, g}-0.93(8) n_{h}-0.09 n_{h} n_{l}+120.75(1) n_{l}-0.82 n_{l}^{2}\right)\left(\frac{\alpha_{s}}{\pi}\right)^{3}+\mathscr{O}\left(\alpha_{s}^{4}\right) .
\end{aligned}
$$

Once the purely gluonic contribution $c_{v, g}$ is available, which is expected to be numerically dominant, $c_{v}$ can be used in the analysis of the third-order cross section $\sigma\left(e^{+} e^{-} \rightarrow t \bar{t}+X\right)$ close to threshold or the extraction of the bottom quark mass from $\Upsilon$ sum rules.

\section{Virtual NNLO corrections to Higgs production in gluon fusion}

In this Section we go beyond the effective theory which has been used in Section 1 in order to define $F_{g}$ and consider the gluon-Higgs vertex with finite top quark mass. This constitutes an important contribution to the gluon fusion process which has the largest production cross section for Higgs bosons both at Tevatron and LHC. Whereas the NLO corrections are exactly known [26, 27, 28 ] at NNLO until recently only approximations for infinitely heavy top quark have been available. Recently this gap has been closed and in the works [29, 30, 31] the NNLO corrections to the cross section $\sigma(p p \rightarrow H+X)$ incorporating the top quark mass dependence have been evaluated. The virtual corrections have been evaluated before in Refs. [32, 33]. In the following we describe in more detail the computation of Ref. [32].

The virtual contribution to the partonic cross section can be cast in the form

$$
\hat{\sigma}_{g g h}^{\mathrm{virt}}=\hat{\sigma}_{\mathrm{LO}}\left(1+\frac{\alpha_{s}}{\pi} \delta^{(1)}+\left(\frac{\alpha_{s}}{\pi}\right)^{2} \delta^{(2)}+\ldots\right),
$$

where the LO cross section is given by $\hat{\sigma}_{\mathrm{LO}}=G_{F} \alpha_{s}^{2} f_{0}(\rho, \varepsilon) \delta(1-x) /(288 \sqrt{2} \pi(1-\varepsilon))$, with $x=M_{H}^{2} / \hat{s}$. $\sqrt{\hat{s}}$ is the partonic center-of-mass energy. The function $f_{0}$ and the analytical results of the first five terms in the $\rho=M_{H}^{2} / M_{t}^{2} \rightarrow 0$ expansion for $\delta^{(1)}$ and $\delta^{(2)}$ can be found in Ref. [32]. Sample diagrams contributing to $\delta^{(2)}$ are shown in Fig. 2(a).

The setup is similar to the one used in Section 2. A difference is connected to the asymptotic expansion in the limit $M_{H}^{2} \ll M_{t}^{2}$ which is performed with the program exp [21] and independently 


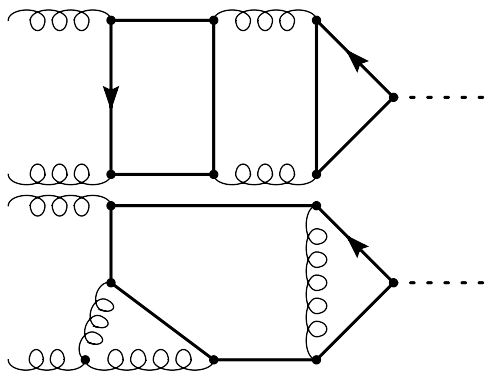

(a)

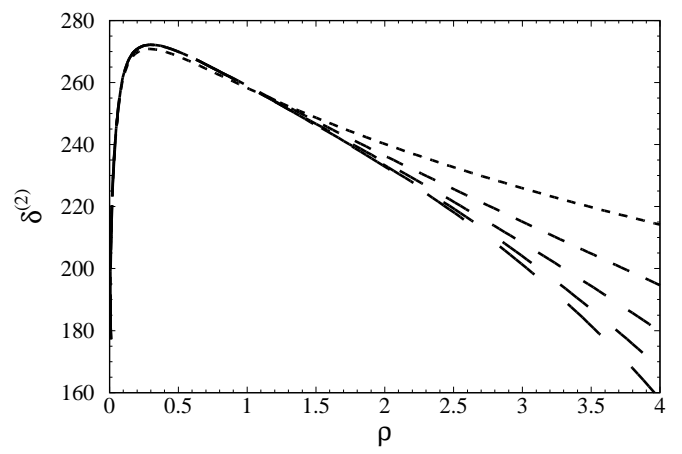

(b)

Figure 2: (a) Sample diagrams contributing to the NNLO virtual corrections to $g g \rightarrow H$. Solid lines represent the top quark with mass $M_{t}$ and curly (dotted) lines massless gluons (the Higgs boson). (b) Finite part of $\delta^{(2)}$ as a function of $\rho$. The longer-dashed lines include successively higher orders in $\rho$.

with an in-house Perl program [34]. In this way the three-loop vertex integrals are reduced to one-, two-, and three-loop vacuum integrals which are treated with MATAD [35], and to massless one- and two-loop vertex contributions which are reduced to master integrals (see, e.g., Ref. [36]) with the help of FIRE [37] and an independent program based of the Laprota-method.

Explicit results for $\delta^{(1)}$ and $\delta^{(2)}$ can be found in Ref. [32]. In this contribution we discuss the convergence properties in Fig. 2(b) where the finite part of $\delta^{(2)}$ is shown as a function of $\rho$. One observes good convergence up to $\rho \approx 3$ which corresponds to $M_{H} \approx 1.7 M_{t} \approx 300 \mathrm{GeV}$.

\section{Acknowledgments}

I would like to thank my colleagues of Refs. [9, 19, 30] for the very fruitful collaboration.

\section{References}

[1] B. Todtli, arXiv:0903.0540 [hep-ph].

[2] P. A. Baikov, Phys. Lett. B 385 (1996) 404, arXiv:hep-ph/9603267; Phys. Lett. B 634, 325 (2006) [arXiv:hep-ph/0507053]; V. A. Smirnov and M. Steinhauser, Nucl. Phys. B 672 (2003) 199 [arXiv:hep-ph/0307088].

[3] P. A. Baikov, PoS RADCOR2007 (2007) 022.

[4] P. A. Baikov, K. G. Chetyrkin and J. H. Kühn, Phys. Rev. Lett. 101 (2008) 012002 arXiv:0801.1821 [hep-ph].

[5] P. A. Baikov and V. A. Smirnov, Phys. Lett. B 477 (2000) 367 [arXiv:hep-ph/0001192].

[6] S. Laporta and E. Remiddi, Phys. Lett. B 379 (1996) 283, arXiv:hep-ph/9602417.

[7] A. V. Smirnov and V. A. Smirnov, JHEP 0601, 001 (2006) [arXiv:hep-lat/0509187].

[8] A. V. Smirnov, JHEP 0810, 107 (2008) [arXiv:0807.3243 [hep-ph]].

[9] P. A. Baikov, K. G. Chetyrkin, A. V. Smirnov, V. A. Smirnov and M. Steinhauser, Phys. Rev. Lett. 102 (2009) 212002 [arXiv:0902.3519 [hep-ph]].

[10] S. Moch, J. A. M. Vermaseren and A. Vogt, JHEP 0508 (2005) 049 [arXiv:hep-ph/0507039]; S. Moch, J. A. M. Vermaseren and A. Vogt, Phys. Lett. B 625 (2005) 245 [arXiv:hep-ph/0508055]. 
[11] G. Heinrich, T. Huber, D. A. Kosower and V. A. Smirnov, Phys. Lett. B 678 (2009) 359 [arXiv:0902.3512 [hep-ph]].

[12] D. J. Broadhurst, N. Gray and K. Schilcher, Z. Phys. C 52 (1991) 111.

[13] K. Melnikov and T. van Ritbergen, Nucl. Phys. B 591 (2000) 515, arXiv:hep-ph/0005131; P. Marquard, L. Mihaila, J. H. Piclum and M. Steinhauser, Nucl. Phys. B 773 (2007) 1, arXiv:hep-ph/0702185.

[14] M. Beneke and V. A. Smirnov, Nucl. Phys. B 522 (1998) 321, arXiv:hep-ph/9711391; V. A. Smirnov, “Applied asymptotic expansions in momenta and masses," Springer Tracts Mod. Phys. 177 (2002) 1.

[15] A. Czarnecki and K. Melnikov, Phys. Rev. Lett. 80 (1998) 2531, arXiv:hep-ph/9712222.

[16] M. Beneke, A. Signer and V. A. Smirnov, Phys. Rev. Lett. 80 (1998) 2535, arXiv:hep-ph/9712302.

[17] B. A. Kniehl, A. Onishchenko, J. H. Piclum and M. Steinhauser, Phys. Lett. B 638 (2006) 209, arXiv:hep-ph/0604072.

[18] P. Marquard, J. H. Piclum, D. Seidel and M. Steinhauser, Nucl. Phys. B 758 (2006) 144, arXiv:hep-ph/0607168.

[19] P. Marquard, J. H. Piclum, D. Seidel and M. Steinhauser, Phys. Lett. B 678 (2009) 269 [arXiv:0904.0920 [hep-ph]].

[20] P. Nogueira, J. Comput. Phys. 105 (1993) 279.

[21] R. Harlander, T. Seidensticker and M. Steinhauser, Phys. Lett. B 426 (1998) 125 , arXiv:hep-ph/9712228; T. Seidensticker, arXiv:hep-ph/9905298.

[22] J. A. M. Vermaseren, arXiv:math-ph/0010025.

[23] P. Marquard and D. Seidel, unpublished.

[24] A. V. Smirnov and M. N. Tentyukov, arXiv:0807.4129 [hep-ph].

[25] A. V. Smirnov, V. A. Smirnov and M. Tentyukov, arXiv:0912.0158 [hep-ph].

[26] S. Dawson, Nucl. Phys. B 359 (1991) 283.

[27] A. Djouadi, M. Spira and P. M. Zerwas, Phys. Lett. B 264 (1991) 440.

[28] M. Spira, A. Djouadi, D. Graudenz and P. M. Zerwas, Nucl. Phys. B 453 (1995) 17, arXiv:hep-ph/9504378.

[29] R. V. Harlander and K. J. Ozeren, JHEP 11 (2009) 088, arXiv:0909.3420 [hep-ph].

[30] A. Pak, M. Rogal and M. Steinhauser, arXiv:0911.4662 [hep-ph].

[31] R. V. Harlander, H. Mantler, S. Marzani and K. J. Ozeren, arXiv:0912.2104 [hep-ph].

[32] A. Pak, M. Rogal and M. Steinhauser, Phys. Lett. B 679 (2009) 473 [arXiv:0907.2998 [hep-ph]].

[33] R. V. Harlander and K. J. Ozeren, Phys. Lett. B 679 (2009) 467 [arXiv:0907.2997 [hep-ph]].

[34] A. Pak, unpublished.

[35] M. Steinhauser, Comput. Phys. Commun. 134 (2001) 335, arXiv:hep-ph/0009029.

[36] T. Gehrmann, T. Huber and D. Maitre, Phys. Lett. B 622 (2005) 295, arXiv:hep-ph/0507061.

[37] A. V. Smirnov, JHEP 0810 (2008) 107, arXiv:0807.3243 [hep-ph]. 